\title{
Research Article \\ Optimal Harvesting When the Exchange Rate Is a Semimartingale
}

\author{
E. R. Offen and E. M. Lungu \\ Department of Mathematics, Faculty of Science, University of Botswana, Private Bag UB 0022, \\ Gaborone, Botswana \\ Correspondence should be addressed to E. R. Offen, elias.offen@gmail.com
}

Received 8 August 2011; Revised 31 October 2011; Accepted 1 November 2011

Academic Editor: Onesimo Hernandez Lerma

Copyright (C 2011 E. R. Offen and E. M. Lungu. This is an open access article distributed under the Creative Commons Attribution License, which permits unrestricted use, distribution, and reproduction in any medium, provided the original work is properly cited.

\begin{abstract}
We consider harvesting in the Black-Scholes Quanto Market when the exchange rate is being modeled by the process $E_{t}=E_{0} \exp \left\{X_{t}\right\}$, where $X_{t}$ is a semimartingale, and we ask the following question: What harvesting strategy $\gamma^{*}$ and the value function $\Phi$ maximize the expected total income of an investment? We formulate a singular stochastic control problem and give sufficient conditions for the existence of an optimal strategy. We found that, if the value function is not too sensitive to changes in the prices of the investments, the problem reduces to that of Lungu and $\varnothing \mathrm{ksendal}$. However, the general solution of this problem still remains elusive.
\end{abstract}

\section{Introduction}

This paper is concerned with an optimal harvesting strategy in the Black-Scholes Quanto Market when the exchange rate is being driven by a general semimartingale. Specifically, it is proposed that the optimal harvesting strategy can be found under certain conditions. The paper aims to make a contribution by deriving the general formula for an optimal harvesting strategy when the exchange rate is a semimartingale. This study could shed light on the application of general semimartingales in optimization of harvests from investments. Optimal harvesting is one of the crucial areas in finance because investment into stocks and bonds can be used as a source of revenue to expand business. Therefore, making investors happy through an optimal harvesting strategy could lead to more investments and consequently to further expansion of business. This study will make reference to dividend policy to illustrate an optimal harvesting strategy. Indeed a suboptimal dividend policy can result in destruction of shareholder confidence. How much to payout and still maintain growth of investments has been a challenge. For example, Miller and Modigilliani [1] claimed that a dividend policy was irrelevant in perfect markets because it had no impact on firm 
value. However, research on dividend policy that followed Miller and Modigilliani [1] has further examined various market imperfections and have identified the relevance of dividend policy. A number of stochastic models for optimal dividend policy can also be found in Taksar [2] and the references therein.

A number of these models $([1,3]$, etc.) have developed an optimal harvesting strategy as optimal stochastic control problems, and that is our approach in this paper. For example, Asmussen and Taksar [4] applied the theory of singular control in their study of a company's optimal dividend policy that tries to maximize expected value of the total (discounted) payments to the shareholders. Asmussen and Taksar [4] made an assumption that no fixed costs are incurred during payment of dividends and that the liquid assets are modeled by Brownian Motion with drift. Others who also contributed to this problem are Højgaard and Taksar [5, 6], Radner and Shepp [7], Asmussen et al. [3], and Choulli et al. [8]. The studies [5-7] treated the problem as a classical singular stochastic control problem but allowed a control to affect both potential profits and the risks of the financial corporation. JeanblancPicqué and Shiryaev [9] investigated the problem of a company that tries to maximize the expected total (discounted) amount of dividend payments by modeling dividends as a stochastic impulse control problem. They [9] looked at a situation whereby the company faced a fixed cost each time a dividend was paid out by choosing optimally the timing and the size of the payments. They [9] assumed that, when there is no intervention, the liquid asset follows a Brownian Motion process with drift. Lungu and Øksendal [10] have considered the problem of optimizing flow of dividends for a market situation with two investments and determined an optimal harvesting strategy. They concluded that the optimal strategy was to do nothing as long as the investments were in the nonintervention region but to harvest when the investment reached a certain calculated value (see Lungu and Øksendal [10]). Motivated by Lungu and Øksendal [10], this study considers a similar problem but now in the BlackScholes Quanto market with the exchange rate modeled by a general semimartingale. The paper is structured as follows. Section 2 states the model and the necessary theory. Section 3 applies the theory, while Section 4 gives the conclusions.

\section{The Model}

In the absence of interventions, the dynamics of $S(t)$, the value of the sterling risky investment, can be modeled by the equation

$$
S_{t}=S_{0} \exp \left\{\alpha t+\sigma W_{t}\right\}
$$

where $\alpha \in \mathbb{R}$ is the riskless interest rate, the constant $\sigma>0$ is the volatility, and $W_{t}$ is Brownian Motion. Let the sterling to dollar exchange be modeled by the equation

$$
E_{t}=E_{0} \exp \left\{X_{t}\right\}
$$

and suppose that these processes are on a filtered probability space $(\Omega, \mathcal{F}, \mathbb{F}, \mathbb{P})$, where $\mathbb{F}=$ $\mathbb{F}^{S} \cup \mathbb{F}^{E}$ and $\mathbb{F}^{S}=\left\{\mathcal{F}_{t}^{S}, t \geq 0\right\}, \mathcal{F}_{t}^{S}=\sigma\left(S_{u}: 0 \leq u \leq t\right)$ is the natural filtration generated by the stock price process while $\mathbb{F}^{E}=\left\{\mathcal{F}_{t}^{E}, t \geq 0\right\}, \mathcal{F}_{t}^{E}=\sigma\left(E_{u}: 0 \leq u \leq t\right)$ is the natural filtration generated by the exchange rate process. $\mathbb{F}$ describes information about prices and the exchange rate revealed to investors. We assume that the probability space $(\Omega, \mathcal{F}, \mathbb{F}, \mathbb{P})$ 
satisfies the usual conditions, that is, the $\sigma$-field $\mathcal{F}$ is $\mathbb{P}$-complete and every $\mathcal{F}_{t}$ contains all $\mathbb{P}$-null sets of $\mathcal{F}$. $X_{t} \in \operatorname{Sem}(\mathbb{F}, \mathbb{P})$, that is, $X_{t}$ is a càdlàg process that admits the decomposition $X_{t}=X_{0}+A_{t}+M_{t}, t \geq 0$, where $A_{t}=A_{t} \in \mho$ (a process of bounded variation), $A_{0}=0$, and $M=M_{t} \in \mathcal{M}_{\text {loc }}$ (a local martingale), $M_{0}=0$.

We consider harvesting from the investment process given (2.1) previously, and we ask the following question: what value function $\Phi(s, y)$ and harvesting strategy $r^{*}(t)$ maximise the total expected discounted utility harvested from a given time interval.

Since our asset is in sterling but our currency of businesses is the dollar, we need first of all to find the dollar equivalence of this asset. To do this, we let $Y_{t}$ be the dollar value of the sterling asset price given by

$$
Y_{t}=E_{t} \cdot S_{t}
$$

Using (2.1) and (2.2), we obtain

$$
\begin{aligned}
Y_{t} & =E_{0} S_{0} \exp \left\{\alpha t+\sigma W_{t}+X_{t}\right\} \\
& =Y_{0} \exp \left\{H_{t}\right\}
\end{aligned}
$$

where

$$
H_{t}=\alpha t+\sigma W_{t}+X_{t}
$$

$H_{t}$ is a semimartingale since it is the sum of two semimartingales $\mu t+\sigma W_{t}$ and $X_{t}$. This in turn implies that $Y_{t}$ is a semimartingale. Our approach will be probabilistic rather than statistical; hence, it becomes reasonable to express $Y_{t}$ in stochastic exponential form.

Theorem 2.1 (Ito's theorem for semimartingales [11]). Let $H_{t}$ be a semimartingale, and let $f$ be a $C^{2}$ real function. Then, $f\left(H_{t}\right)$ is again a semimartingale and

$$
f\left(H_{t}\right)=f\left(H_{0}\right)+\int_{0}^{t} f^{\prime}\left(H_{s^{-}}\right) d H_{s}+\frac{1}{2} \int_{0}^{t} f^{\prime \prime}\left(H_{s-}\right) d\left\langle H^{c}\right\rangle_{s}+\sum_{0 \leq s \leq t}\left[f\left(H_{s}\right)-f\left(H_{s^{-}}\right)-f^{\prime}\left(H_{s^{-}}\right) \Delta H_{s}\right]
$$

where $\Delta H_{s}=H_{s}-H_{s-}$ and $\left\langle H^{c}\right\rangle_{t}$ is the quadratic characteristic of the continuous martingale part $H_{t}^{c}$ of $H_{t}$, that is, a predictable process such that $\left(H^{c}\right)_{t}^{2}-\left\langle H^{c}\right\rangle_{t} \in \mathcal{M}_{\mathrm{loc}}$.

For the proof of Theorem 2.1, the reader is referred to Protter [11].

In this study, we use Theorem 2.1 to rewrite $Y_{t}$ in stochastic exponential form.

Let

$$
Y_{t}=f\left(H_{t}\right)=Y_{0} \exp \left(H_{t}\right)
$$


then, using Theorem 2.1, we have

$$
\begin{aligned}
Y_{t} & =Y_{0}\left[e^{H_{0}}+\int_{0}^{t}\left(e^{H_{s-}}\right)^{\prime} d H_{s}+\frac{1}{2} \int_{0}^{t}\left(e^{H_{s-}}\right)^{\prime \prime} d\left\langle H^{c}\right\rangle_{s}+\sum_{0 \leq s \leq t}\left(e^{H_{s}}-e^{H_{s-}}-\left(e^{H_{s-}}\right)^{\prime} \Delta H_{s}\right)\right] \\
& =Y_{0}\left[e^{H_{0}}+\int_{0}^{t} e^{H_{s-}} d H_{s}+\frac{1}{2} \int_{0}^{t} e^{H_{s-}} d\left\langle H^{c}\right\rangle_{s}+\sum_{0 \leq s \leq t}\left(e^{H_{s}}-e^{H_{s-}}-e^{H_{s-}} \Delta H_{s}\right)\right]
\end{aligned}
$$

and, in differential form, this can be expressed as

$$
\begin{aligned}
d Y_{t} & =Y_{0}\left[e^{H_{t-}} d H_{t}+\frac{1}{2} e^{H_{t-}} d\left\langle H^{c}\right\rangle_{t}+e^{H_{t}}-e^{H_{t-}}-e^{H_{t-}} \Delta H_{t}\right] \\
& =Y_{0}\left[e^{H_{t-}} d H_{t}+\frac{1}{2} e^{H_{t-}} d\left\langle H^{c}\right\rangle_{t}+e^{H_{t-}+\Delta H_{t}}-e^{H_{t-}}-e^{H_{t-}} \Delta H_{t}\right] \\
& =e^{H_{t-}} d H_{t}+\frac{1}{2} e^{H_{t-}} d\left\langle H^{c}\right\rangle_{t}+e^{H_{t-}}\left(e^{\Delta H_{t}}-1-\Delta H_{t}\right) \\
& =e^{H_{t-}} d\left[H_{t}+\frac{1}{2}\left\langle H^{c}\right\rangle_{t}+\sum_{0<s \leq t}\left(e^{\Delta H_{s}}-1-\Delta H_{s}\right)\right] \\
& =Y_{t-} d \widehat{H}_{t},
\end{aligned}
$$

where

$$
\widehat{H}_{t}=H_{t}+\frac{1}{2}\left\langle H^{c}\right\rangle_{t}+\sum_{0<s \leq t}\left(e^{\Delta H_{s}}-1-\Delta H_{s}\right)
$$

We associate with this semimartingale $Y_{t}$ an integer-valued random measure defined as

$$
\mu_{n}(A ; \omega)=I_{A}\left(\Delta X_{n}(\omega)\right), \quad A \in \mathbb{B}\left(\mathbb{R}^{d}\right)
$$

where $I$ is an indicator function, that is,

$$
\mu_{n}(A ; \omega)= \begin{cases}1 & \text { if } \Delta X_{n}(\omega) \in A, \\ 0 & \text { if } \Delta X_{n}(\omega) \notin A,\end{cases}
$$

(see Shiryaev [12], for details). We define the integral-valued random measures of jumps $\mu^{X}=\left(\mu_{(0, n]}^{X}(\cdot)\right)_{n \geq 1}$, where $\mu_{(0, n]}^{X}(A ; \omega)=\sum_{k=1}^{n} \mu_{k}^{X}(A, \omega)$ and $\mu_{k}^{X}(A ; \omega)=I\left(\Delta X_{k}(\omega) \in A, A \in\right.$ $B\left(\mathbb{R}^{d} \backslash\{0\}\right)$ and by $v=v(\omega, d s, d x)$ the compensator of $\mu$, that is, the predictable measure $v_{n}(A: \omega)=\mathbb{P}\left(\Delta X_{n} \in A \mid \mathcal{F}_{n-1}\right)(\omega)(\mathbb{P}$-a.s. $)$ with the property that $\mu-v$ is a local martingale measure. This means that, for each $A \in \mathbb{B}(\mathbb{R} \backslash\{0\}),(\mu(w:(0, t] \times A)-v(\omega ;(0, t] \times A))_{t>0}$ is a local martingale with value 0 for $t=0$. 
Let $\varphi$ be a truncation function, for example, $\varphi(x)=x I(|x| \leq 1)$. Then $\Delta X_{s}-$ $\varphi\left(\Delta X_{s}\right) \neq 0 \Leftrightarrow\left|\Delta X_{s}\right|>\alpha$ for some $\alpha>0$. We now denote the jump part of $X$ corresponding to big jumps by

$$
\check{X}=\sum_{0<k \leq t}\left(\left(\Delta X_{k}\right)-\varphi\left(\Delta X_{k}\right)\right)
$$

Using random measure of jumps, (2.9) can be written in canonical form as

$$
\begin{aligned}
& d Y_{t}=Y_{t-} d H_{t} \\
& =Y_{-}\left(\alpha d t+d C(\varphi)_{t}+\frac{1}{2} d\left\langle H^{c}\right\rangle+\sigma d W_{t}+d X_{t}^{c}+d\left(e^{x}-1\right) \star(\mu-v)\right. \\
& \left.\quad+d\left(e^{x}-1-\varphi(x)\right) \star \mu\right),
\end{aligned}
$$

where $C(\varphi)$ is a predictable process and $X^{c}$ is the continuous martingale part of $X_{t}$ [13].

Adopting the definition of harvesting strategy from Lungu and Øksendal [10], we define a harvesting strategy as a stochastic process with the following properties: $\gamma(t, \omega) \in$ $\mathbb{R}, t>s, \omega \in \Omega$,

(1) $\gamma(t)$ is measurable with respect to $\sigma$-algebra $\mathcal{F}_{t}$ generated by $X(s, \cdot) ; s \leq t$ (i.e., $\{\gamma(t)\}_{\gamma \geq 0}$ is adapted);

(2) $\gamma(t, \omega)$ is nondecreasing with respect to $t$ for almost all (a.a.) $\omega$;

(3) $\gamma(t, \omega)$ is right continuous as a function of $t$ for a.a. $\omega$;

(4) $\gamma(s, \omega)=0$ for a.a. $\omega$.

$\gamma(t, \omega)$ represents the total amount harvested from an initial time $s$ up to time $t$. We let $\Gamma$ represent a set of all harvesting strategies. If we apply the harvesting strategy $r(t, \omega)$, then the corresponding process $Y^{r}$ satisfies the equation

$$
\begin{aligned}
d Y_{t}^{\gamma=} & Y_{-}^{\gamma}\left(\alpha d t+d C(\varphi)_{t}+\frac{1}{2} d\left\langle H^{c}\right\rangle_{t}+\sigma d W_{t}+d X_{t}^{c}+d\left(e^{x}-1\right) \star(\mu-v)\right. \\
& \left.+d\left(e^{x}-1-\phi(x) \star \mu\right)\right)-d \gamma(t), \\
Y^{\gamma}\left(s^{-}\right)= & y_{1} .
\end{aligned}
$$

It is important to note that the difference between $Y^{\gamma}(s)$ and $Y^{\gamma}\left(s^{-}\right): Y^{\gamma}\left(s^{-}\right)$is the state before harvesting starts at time $t=s$ while $Y^{\gamma}(s)$ is the state immediately after. If $\gamma$ consists of an immediate harvest of size $\gamma$ at $t=s$, then

$$
Y(s)=Y\left(s^{-}\right)-\Delta \gamma
$$


Let the prices/utilities per unit investment when harvested at time $t$ be given by a constant nonnegative function $\pi$. The expected total discounted payoff in this case is given by

$$
J^{\gamma}(s, y)=\mathbb{E}^{y}\left[\int_{0}^{T} \pi e^{-\rho(s+t)} \cdot d \gamma(t)\right]
$$

where $\mathbb{E}^{s, y}$ denotes expectation with respect to probability law $Q^{s, y}$ of $Y^{s, y}(t)=\left(t, Y^{\gamma}(t)\right)$ for $t \geq s$, assuming that $Y^{s, y}(0)=(s, y)$,

$$
\begin{gathered}
T=\inf \{t>0 ; \pi Y(t) \notin S\}, \\
S=\left\{(s, y): \pi e^{-\rho(s+t)} y \geq 0\right\}
\end{gathered}
$$

are the time of bankruptcy and $S$ is the solvency region, respectively. The optimal harvesting problem is then to find the value function $\Phi(s, y)$ and an optimal dividend strategy $\gamma^{*}(t)$ such that

$$
\Phi(s, y)=\sup _{r \in \Gamma} J^{r}(s, y)=J^{r^{*}}(s, y)
$$

We let $0<t_{1}<t_{2} \cdots$ denote the jumping times of the given strategy $\gamma \in \Gamma$, and we let $\Delta\left(t_{k}\right)=\gamma\left(t_{k}\right)-\gamma\left(t_{k}^{-}\right)$be the jump of $\gamma\left(t_{k}\right)$. We also let

$$
\gamma^{c}(t)=\gamma(t)-\sum_{s \leq t_{k} \leq t} \Delta \gamma\left(t_{k}\right)
$$

be the continuous part of $\gamma(t)$. We formulate the sufficient conditions for the given function $\phi(s, y)$ to be the value function $\Phi(s, y)$ of (2.19) and for a given strategy $\widehat{\gamma} \in \Gamma$ to be optimal in the following theorem.

Theorem 2.2 (extended Lungu and Øksendal [10]). Suppose that $\phi \geq 0$ is twice continuously differentiable on $S$ with the following properties:

(1) One has

$$
\frac{\partial \phi}{\partial y} \geq \pi e^{-\rho(s+t)}
$$

(2) One has

$$
\begin{aligned}
L \phi(t, y) & =\frac{\partial \phi}{\partial t}+\left(\alpha t+C(\varphi)_{t}+\frac{1}{2}\left(\sigma^{2}+\left\langle H^{c}\right\rangle_{t}\right)+\left(e^{y}-1\right) \star \mu\right) \frac{\partial \phi}{\partial y}+\frac{1}{2} \sigma^{2} \frac{\partial \phi^{2}}{\partial y^{2}} \\
& \leq 0 \quad \text { on } S .
\end{aligned}
$$


International Journal of Stochastic Analysis

Then

$$
\phi(s, y) \geq \Phi(s, y) \quad \text { on } S
$$

(3) Define the nonintervention region as

$$
D=\left\{(t, y) \in S ; \frac{\partial \phi}{\partial y}(t, y)>\pi e^{-\rho(s+t)} \forall i=1 \cdots n\right\}
$$

Suppose

$$
L \phi=0 \quad \text { in } D,
$$

and there exists a harvesting strategy $\hat{\gamma}$ such that the following hold:

$$
\begin{gathered}
\Upsilon^{\widehat{\gamma}}(t) \in \bar{D} \quad \forall t>s, \\
\left(\frac{\partial \phi}{\partial y}\left(t, Y^{\widehat{\gamma}}(t)\right)-\pi e^{-\rho(s+t)}\right) \widehat{\gamma}^{c}(t)=0 \quad \forall i=1 \cdots n, \\
\quad\left(\text { i.e., } \widehat{\gamma}^{c} \text { increases only when } \frac{\partial \phi}{\partial y}=\pi e^{-\rho(s+t)}\right),
\end{gathered}
$$

where $\bar{D}$ is the closure of $D$, that is, $\bar{D}=D \cup \partial D$, where $\partial D$ is the boundary of $D$.

(4) One has

$$
\begin{aligned}
\frac{\partial \phi}{\partial y}\left(t, Y^{\widehat{\gamma}}(s)\right) & \left(\alpha d t+d C(\varphi)_{t}+\frac{1}{2} d\left\langle H^{c}\right\rangle+\int_{-\infty}^{\infty}\left(e^{y}-1-\varphi(y) d \mu\right)\right) \\
& -\left(\alpha t+C(\varphi)_{t}+\frac{1}{2}\left(\sigma^{2}+\left\langle H^{c}\right\rangle_{t}\right)+\int_{-\infty}^{\infty}\left(e^{y}-1-\varphi(y)\right) d \mu\right) d t \leq 0
\end{aligned}
$$

(5) One has

$$
\Delta \phi\left(t_{k}\right):=\phi\left(t_{k}, Y^{\widehat{\gamma}}\left(t_{k}\right)\right)-\phi\left(t_{k}, Y^{\widehat{\gamma}}\left(t_{k}^{-}\right)\right)=-\pi \cdot \Delta \widehat{\gamma}\left(t_{k}\right)
$$

at all jumping times $t_{k} \geq s$ of $\widehat{\gamma}\left(t_{k}\right)$ and

$$
\mathbb{E}^{s, y}\left[\phi\left(T_{R}, Y^{\widehat{\gamma}}\left(T_{R}\right)\right)\right] \longrightarrow \infty
$$

where

$$
T_{R}=T \wedge R \wedge \inf \left\{t>s ;\left|Y^{\widehat{r}}(t)\right| \geq R\right\}
$$


Then

$$
\phi(s, y)=\Phi(s, y) \quad \forall(s, y) \in S
$$

and $\gamma=\gamma^{*}$ is an optimal harvest strategy.

Proof. Consider the following:

$$
\begin{aligned}
& \Delta Y_{t}=\alpha \Delta t+\Delta C(\varphi)_{t}+\Delta\left\langle H^{c}\right\rangle_{t}+\sigma \Delta W_{t}+\Delta X_{t}^{c}+\Delta\left(e^{x}-1\right) \star(\mu-v)+\Delta\left(e^{x}-1-\varphi\right) \star v \\
& =\Delta\left(e^{x}-1\right) \star(\mu-v)+\Delta\left(e^{x}-1-\varphi\right) \star v, \\
& d Y_{t}=\alpha d t+d C(\varphi)_{t}+\frac{1}{2} d\left\langle H^{c}\right\rangle+\sigma d W_{t}+d X_{t}^{c}+d\left(e^{y}-1\right) \star(\mu-v) \\
& +d\left(e^{x}-1-\varphi(x) \star \mu\right)-d \gamma(t) \\
& =\alpha d t+d C(\varphi)_{t}+\frac{1}{2} d\left\langle H^{c}\right\rangle+\sigma d W_{t}+d X_{t}^{c}+\int_{-\infty}^{\infty}\left(e^{x}-1-\varphi(y)\right) d(\mu-v) \\
& +\int_{-\infty}^{\infty}\left(e^{x}-1-\varphi(y)\right) d \mu-d \gamma(t)
\end{aligned}
$$

Choose $\gamma \in \Gamma$, and assume that $\phi \in C^{2}$ satisfies (2.21)-(2.22). Then, by Ito's formula for semimartingales and then computing the expectation throughout the equation

$$
\begin{aligned}
\mathbb{E}^{s y}\left[\phi\left(T_{R}, Y^{\gamma}\left(T_{R}\right)\right)\right] & \\
= & \mathbb{E}^{s, y}\left[\phi\left(s, Y^{\gamma}(s)\right)\right]+\mathbb{E}^{s, y}\left[\int_{s+}^{T_{R}} \frac{\partial \phi}{\partial t}\left(t, Y^{\gamma}(s)\right) d t\right] \\
+ & \mathbb{E}^{s, y}\left[\int _ { s + } ^ { T _ { R } } \left\{\frac { \partial \phi } { \partial y } ( t , Y ^ { \gamma } ( s ) ) \left(\alpha d t+d C(\varphi)_{t}+\frac{1}{2} d\left\langle H^{c}\right\rangle_{t}+\sigma d W_{t}+d X_{t}^{c}\right.\right.\right. \\
& \left.\left.\left.+\int_{-\infty}^{\infty}\left(e^{x}-1\right) d(\mu-v)+\int_{-\infty}^{\infty}\left(e^{x}-1-\varphi(y)\right) d \mu\right)-d \gamma(t)\right\}\right] \\
+ & \mathbb{E}^{s, y}\left[\int_{s+}^{T_{R}} \frac{1}{2} \sigma^{2}\left(t, Y^{\gamma}(t)\right) \frac{\partial^{2} \phi}{\partial y^{2}}\left(t, Y^{\gamma}\right)\right] \\
+ & \mathbb{E}^{s, y}\left[\sum_{s<t_{k} \leq T_{R}}\left\{\phi\left(t_{k}, Y^{\gamma}\left(t_{k}\right)\right)-\phi\left(t_{k}, Y^{\gamma}\left(t_{t_{k}}^{-}\right)\right)-\frac{\partial \phi}{\partial y}\left(t_{k}, Y^{\phi}\left(t_{k}\right)\right) \Delta Y_{i}^{\gamma}\left(t_{k}\right)\right\}\right] .
\end{aligned}
$$

From the theory of martingales for integrals,

$$
\mathbb{E}^{s, y}\left[\int_{s+}^{T_{R}} \sigma \frac{\partial \phi}{\partial y} d W_{t}\right]=\mathbb{E}^{s, y}\left[\int_{s+}^{T_{R}} \sigma \frac{\partial \phi}{\partial y} d X_{t}^{c}\right]=\mathbb{E}^{s, y}\left[\int_{s^{+}}^{T_{R}} \int_{-\infty}^{\infty}\left(e^{y}-1\right) d(\mu-v)\right]=0 .
$$


Substituting (2.28), (2.32), and (2.35) in (2.34), we have

$$
\begin{aligned}
\mathbb{E}^{s y} & {\left[\phi\left(T_{R}, Y^{\gamma}\left(T_{R}\right)\right)\right] } \\
= & \mathbb{E}^{s y}[\phi(s, y)]+\mathbb{E}^{s, y}\left[\int_{s+}^{T_{R}} \frac{\partial \phi}{\partial t}\left(t, Y^{\gamma}(s)\right) d t\right] \\
+ & \mathbb{E}^{s, y}\left[\int_{s^{+}}^{T_{R}}\left\{\frac{\partial \phi}{\partial y}\left(t, Y^{\gamma}(s)\right)\left(\alpha d t+d C(\phi)_{t}+\frac{1}{2} d\left\langle H^{c}\right\rangle_{t}+\int_{-\infty}^{\infty}\left(e^{x}-1-\varphi(x)\right) d \mu\right)-d \gamma(t)\right\}\right] \\
+ & \mathbb{E}^{s, y}\left[\int_{s^{+}}^{T_{R}} \frac{1}{2} \sigma^{2}\left(t, X^{\gamma}(t)\right) \frac{\partial^{2} \phi}{\partial y^{2}}\left(t, Y^{\gamma}\right)\right] \\
+ & \mathbb{E}^{s, y}\left[\sum_{s<t_{k} \leq T_{R}}\left\{\left(\Delta \phi\left(t_{k}, Y^{\gamma}\left(t_{k}\right)\right)-\frac{\partial \phi}{\partial y}\left(t_{k}, Y^{\phi}\left(t_{k}\right)\right)\left(\Delta\left(e^{x}-1\right) \star(\mu-v)+\Delta\left(e^{x}-1-\varphi\right) \star v\right)\right)\right\}\right] .
\end{aligned}
$$

Now from (2.22),

$$
\begin{aligned}
\frac{\partial \phi}{\partial t}= & L \phi(t, x)-\left(\alpha t+C(\varphi)_{t}+\frac{1}{2}\left(\sigma^{2}+\left\langle H^{c}\right\rangle_{t}\right)+\left(e^{x}-1-\varphi(y)\right) \star \mu\right) \frac{\partial \phi}{\partial y} \\
& -\frac{1}{2} \sigma^{2}(t, x) \frac{\partial^{2} \phi}{\partial y^{2}}
\end{aligned}
$$

Using (2.37) in (2.36) gives

$$
\begin{aligned}
& \mathbb{E}^{s y}\left[\phi\left(T_{R}, Y^{\gamma}\right)\right] \\
& =\mathbb{E}^{s y}\left[\phi\left(T_{R}, Y^{\gamma}\right)\right] \\
& +\mathbb{E}^{s, y}\left[\int_{s^{+}}^{T_{R}} L \phi(t, x) d t\right] \\
& +\mathbb{E}^{s, y}\left[\int _ { s ^ { + } } ^ { T _ { R } } \left\{\frac { \partial \phi } { \partial y } \left(t, Y^{\gamma}(s)\left(\alpha d t+d C(\varphi)_{t}+\frac{1}{2} d\left\langle H^{c}\right\rangle+\int_{-\infty}^{\infty}\left(e^{y}-1-\varphi(y) d \mu\right)\right)\right.\right.\right. \\
& \left.\quad-\left(\alpha t+C(\varphi)_{t}+\frac{1}{2}\left(\sigma^{2}+\left\langle H^{c}\right\rangle_{t}\right)+\int_{-\infty}^{\infty}\left(e^{y}-1-\varphi(y)\right) d \mu d t\right\}\right] \\
& +\mathbb{E}^{s, y}\left[\int_{s+}^{T_{R}} \frac{\partial \phi}{\partial y}\left(t, Y^{\gamma}(s)\right) d \gamma(t)\right] \\
& +\mathbb{E}^{s x}\left[\sum_{s<t_{k} \leq T_{R}}\left\{\left(\Delta \phi\left(t_{k}, Y^{\gamma}\left(t_{k}\right)\right)-\frac{\partial \phi}{\partial y}\left(t_{k}, Y^{\phi}\left(t_{k}\right)\right)\left(\Delta\left(e^{x}-1\right) \star(\mu-v)+\Delta\left(e^{x}-1-\varphi\right) \star v\right)\right)\right\}\right]
\end{aligned}
$$


Using the fact that $\mathbb{E}^{s y}\left[\phi\left(T_{R}, Y^{\gamma}\right)\right] \leq \phi(s, x)$ and that $L \phi(s, x) \leq 0$ (refer to (2.27)) yields the inequality

$$
\begin{aligned}
& \mathbb{E}^{s y}\left[\phi\left(T_{R}, Y^{\gamma}\right)\right] \\
& \leq \phi(s, y)-\mathbb{E}^{s, y}\left[\int_{s+}^{T_{R}} \frac{\partial \phi}{\partial y}\left(t, Y^{\gamma}(s)\right) d \gamma(t)\right] \\
& \quad+\mathbb{E}^{s x}\left[\sum_{s<t_{k} \leq T_{R}}\left\{\left(\Delta \phi\left(t_{k}, Y^{\gamma}\left(t_{k}\right)\right)-\frac{\partial \phi}{\partial y}\left(t_{k}, Y^{\phi}\left(t_{k}\right)\right)\left(\Delta\left(e^{x}-1\right) \star(\mu-v)+\Delta\left(e^{x}-1-\phi\right) \star v\right)\right)\right\}\right] .
\end{aligned}
$$

From the relation

$$
r^{c}(t)=\gamma(t)-\sum_{s \leq t_{k} \leq t} \Delta \gamma\left(t_{k}\right)
$$

we deduce that

$$
\begin{aligned}
d \gamma(t) & =d \gamma^{c}(t)+\Delta(t) \\
\Delta Y(t) & =-\Delta \gamma(t)
\end{aligned}
$$

Using (2.41), the right-hand side of (2.32) becomes

$$
\Delta\left(e^{x}-1\right) \star(\mu-v)+\Delta\left(e^{x}-1-\phi\right) \star v=-\Delta \gamma(t)
$$

Furthermore, using (2.42) we can achieve the simplification of (2.34) as follows: taking the last three terms in (2.39) and using the fact that

$$
\sum_{s \leq t_{k} \leq T_{R}} f\left(t_{k}\right)=\sum_{s<t_{k} \leq T_{R}} f\left(t_{k}\right)+\Delta f(s),
$$

we have

$$
\begin{aligned}
& \mathbb{E}^{s y}\left[\sum_{s<t_{k} \leq T_{R}}\left\{\left(\Delta \phi\left(t_{k}, Y^{\gamma}\left(t_{k}\right)\right)-\frac{\partial \phi}{\partial y}\left(t_{k}, Y^{\phi}\left(t_{k}\right)\right)\left(\Delta\left(e^{x}-1\right) \star(\mu-v)+\Delta\left(e^{x}-1-\phi\right) \star v\right)\right)\right\}\right] \\
= & \mathbb{E}^{s y}\left[\sum_{s \leq t_{k} \leq T_{R}}\left\{\left(\Delta \phi\left(t_{k}, Y^{\gamma}\left(t_{k}\right)\right)-\frac{\partial \phi}{\partial y}\left(t_{k}, Y^{\phi}\left(t_{k}\right)\right)\left(\Delta\left(e^{x}-1\right) \star(\mu-v)+\Delta\left(e^{x}-1-\phi\right) \star v\right)\right)\right\}\right] \\
& -\mathbb{E}^{s, y}\left[\Delta \phi\left(s, Y^{\gamma}(s)\right)\right]-\mathbb{E}^{s, y}\left[\frac{\partial \phi}{\partial y}\left(s, Y^{\phi}(s)\right)\left(\Delta\left(e^{x}-1\right) \star(\mu-v)+\Delta\left(e^{x}-1-\phi\right) \star v\right)\right] \\
= & \mathbb{E}^{s y}\left[\sum_{s \leq t_{k} \leq T_{R}}\left\{\Delta \phi\left(t_{k}, Y^{\gamma}\left(t_{k}\right)\right)-\frac{\partial \phi}{\partial y}\left(t_{k}, Y^{\gamma}\left(t_{k}\right)\right) \Delta \gamma(t)\right\}\right] \\
& -\mathbb{E}^{s, y}\left[\Delta \phi\left(s, Y^{\gamma}(s)\right)\right]+\mathbb{E}^{s, y}\left[\frac{\partial \phi}{\partial y}\left(s, Y^{\gamma}(s)\right) \Delta \gamma(s)\right] .
\end{aligned}
$$


Substituting (2.44) into (2.39), inequality (2.39) becomes

$$
\begin{aligned}
\mathbb{E}^{s y}\left[\phi\left(T_{R}, Y^{\gamma}\right)\right] \leq & \phi(s, y)-\mathbb{E}^{s, y}\left[\int_{s+}^{T_{R}} \frac{\partial \phi}{\partial y}\left(t, Y^{\gamma}(s)\right) d \gamma(t)\right]-\mathbb{E}^{s, y}\left[\sum_{s \leq t_{k} \leq T_{R}} \frac{\partial \phi}{\partial y}\left(t, Y^{\gamma}(s)\right) \Delta \gamma(t)\right] \\
& +\mathbb{E}^{s y}\left[\sum_{s \leq t_{k} \leq T_{R}} \Delta \phi\left(t_{k}, Y^{\gamma}\left(t_{k}\right)\right)\right]+\mathbb{E}^{s y}\left[\sum_{s \leq<t_{k} \leq T_{R}} \frac{\partial \phi}{\partial y}\left(t_{k}, Y^{\phi}\left(t_{k}\right)\right) \Delta \gamma(t)\right] \\
& -\mathbb{E}^{s, y}\left[\Delta \phi\left(s, Y^{\gamma}(s)\right)\right]+\mathbb{E}^{s, y}\left[\frac{\partial \phi}{\partial y}\left(s, Y^{\gamma}(s)\right) \Delta \gamma(s)\right] .
\end{aligned}
$$

This will then lead to the inequality

$$
\begin{aligned}
\mathbb{E}^{s y}\left[\phi\left(T_{R}, Y^{\gamma}\left(T_{R}\right)\right)\right] \leq & \phi(s, y)-\mathbb{E}^{s, y}\left[\int_{s+}^{T_{R}} \frac{\partial \phi}{\partial y}\left(t, Y^{\gamma}(s)\right) d \gamma^{c}(t)\right] \\
& +\mathbb{E}^{s y}\left[\sum_{s \leq t_{k} \leq T_{R}} \Delta \phi\left(t_{k}, Y^{\gamma}\left(t_{k}\right)\right)\right] .
\end{aligned}
$$

By the Mean-Value property, we have

$$
\Delta \phi\left(t_{k}, \widehat{Y}^{\gamma}\left(t_{k}\right)\right)=\frac{\partial \phi}{\partial y}\left(t_{k}, \widehat{Y}_{k}^{\gamma}\right) \Delta \widehat{Y}_{i}^{\gamma}\left(t_{k}\right)
$$

for some point $\widehat{Y}_{k}^{\gamma}$ on the line connecting the points $Y^{\gamma}\left(t_{k}^{-}\right)$and $Y^{\gamma}\left(t_{k}\right)$, and, using (2.41), we have

$$
\Delta \phi\left(t_{k}, \hat{y}^{\gamma}\left(t_{k}\right)\right)=\frac{\partial \phi}{\partial y}\left(t_{k}, \hat{Y}_{k}^{\gamma}\right) \Delta \widehat{Y}_{i}^{\gamma}\left(t_{k}\right)=-\frac{\partial \phi}{\partial y}\left(t_{k}, \hat{X}_{(k)}^{\gamma}\right) \Delta \gamma\left(t_{(k)}\right)
$$

which leads to

$$
\begin{aligned}
\mathbb{E}^{s y}\left[\phi\left(T_{R}, Y^{\gamma}\left(T_{R}\right)\right)\right] \leq & \phi(s, y)-\mathbb{E}^{s, y}\left[\int_{s+}^{T_{R}} \frac{\partial \phi}{\partial y}\left(t, Y^{\gamma}(s)\right) d \gamma^{c}(t)\right] \\
& -\mathbb{E}^{s y}\left[\sum_{s \leq t_{k} \leq T_{R}} \frac{\partial \phi}{\partial y}\left(t_{k}, \hat{X}_{(k)}^{\gamma}\right) \Delta \gamma\left(t_{(k)}\right)\right] .
\end{aligned}
$$

From (2.21), we obtain

$$
\phi(s, y)-\int_{s+}^{T_{R}} \frac{\partial \phi}{\partial y}\left(t, Y^{\gamma}(s)\right) d \gamma^{c}(t) \leq \phi(s, x)-\int_{s+}^{T_{R}} \pi \cdot e^{-(\rho+s) t} d \gamma^{c}(t)
$$


Equation (2.21) can also be expressed in discrete form as

$$
\sum_{s \leq t_{k} \leq T_{R}} \frac{\partial \phi}{\partial y}\left(t_{k}, \hat{X}_{(k)}^{\gamma}\right) \Delta \gamma\left(t_{(k)}\right) \geq \sum_{s \leq t_{k} \leq T_{R}} \pi e^{-(\rho+s) t} \Delta \gamma\left(t_{(k)}\right)
$$

Combining (2.50) and (2.51) gives the inequality

$$
\begin{aligned}
\phi(s, y) & -\int_{s+}^{T_{R}} \frac{\partial \phi}{\partial y}\left(t, Y^{\gamma}(s)\right) d \gamma^{c}(t)-\sum_{s \leq t_{k} \leq T_{R}} \frac{\partial \phi}{\partial y}\left(t_{k}, \hat{Y}_{(k)}^{\gamma}\right) \Delta \gamma\left(t_{(k)}\right) \\
& \leq \phi(s, y)-\int_{s+}^{T_{R}} \pi \cdot e^{-(\rho+s) t} d \gamma^{c}(t)-\sum_{s \leq t_{k} \leq T_{R}} \pi e^{-(\rho+s) t} \Delta \gamma\left(t_{(k)}\right) .
\end{aligned}
$$

Taking the expectation of (2.52) yields the inequality

$$
\begin{aligned}
\mathbb{E}^{s, y}\left[\phi\left(T_{R}, Y^{\gamma}\left(T_{R}\right)\right)\right] \leq & \phi(s, y)-\mathbb{E}^{s, y}\left[\int_{s+}^{T_{R}} \frac{\partial \phi}{\partial y}\left(t, Y^{\gamma}(s)\right) d \gamma^{c}(t)\right] \\
& -\mathbb{E}^{s y}\left[\sum_{s \leq t_{k} \leq T_{R}} \frac{\partial \phi}{\partial y}\left(t_{k}, \widehat{X}_{(k)}^{\gamma}\right) \Delta \gamma\left(t_{(k)}\right)\right] \\
\leq & \phi(s, y)-\mathbb{E}^{s y}\left[\int_{s+}^{T_{R}} \pi \cdot e^{-(\rho+s) t} d \gamma^{c}(t)\right]-\mathbb{E}^{s, y}\left[\sum_{s \leq t_{k} \leq T_{R}} \pi e^{-(\rho+s) t} \Delta \gamma\left(t_{(k)}\right)\right],
\end{aligned}
$$

from which we obtain

$$
\begin{aligned}
\phi(s, y) & \geq \mathbb{E}^{s, y}\left[\int_{s+}^{T_{R}} \pi \cdot e^{-(\rho+s) t} d \gamma^{c}(t)\right]+\mathbb{E}^{s y}\left[\sum_{s \leq t_{k} \leq T_{R}} \pi e^{-(\rho+s) t} \Delta \gamma\left(t_{(k)}\right)\right]+\mathbb{E}^{s y}\left[\phi\left(T_{R}, Y^{\gamma}\left(T_{R}\right)\right)\right] \\
& \geq \mathbb{E}^{s y}\left[\int_{s}^{T_{R}} \pi \cdot e^{-(\rho+s) t} d \gamma(t)\right]+\mathbb{E}^{s y}\left[\phi\left(T_{R}, Y^{\gamma}\left(T_{R}\right)\right)\right] .
\end{aligned}
$$

Since $R<\infty, \gamma \in \Gamma$ were arbitrary and $\phi \geq 0$, this proves that

$$
\phi(s, y) \geq \Phi(s, y) \text { and } \hat{\gamma} \text { is optimal. }
$$


Let us now assume that $D$ is given by (2.24) and that (2.25)-(2.28) hold. If we replace $\gamma$ in the above calculation by $\hat{\gamma}$, then equality holds everywhere and we end up with the relation

$$
\phi(s, y)=\mathbb{E}^{s, y}\left[\int_{s}^{T_{R}} \pi \cdot e^{-(\rho+s) t} d \widehat{\gamma}+\mathbb{E} \phi\left(T_{R}, Y^{\widehat{\gamma}}\left(T_{R}\right)\right)\right] .
$$

Letting $R \rightarrow \infty$ and using (2.29), we get

$$
\phi(s, y)=\mathbb{E}^{s, y}\left[\int_{s}^{\infty} \pi \cdot e^{-(\rho+s) t} d \widehat{\gamma}\right]
$$

Combining (eqntawina) with (2.23), we have

$$
\phi(s, y)=\Phi(s, y), \quad \hat{\gamma} \text { is optimal. }
$$

The strategy $\hat{\gamma}$ can be found by solving the Skorohod stochastic differential equations (see Lungu and Øksendal [10]).

\section{Application of the Theory}

From the form of our discounted utility function (2.17), it becomes reasonable to look for the function $\Phi$ of the form

$$
\Phi(s, y)=e^{-\rho s} \Psi(y)
$$

Let

$$
\phi(s, y)=e^{-\rho s} \psi(y)
$$

Equations (2.21), (2.22) become

$$
\begin{aligned}
\frac{\partial \psi}{\partial y} \geq & \pi \\
L \psi(t, y)= & -\rho \psi+\left(\alpha t+C(\varphi)_{t}+\frac{1}{2}\left(\sigma^{2}+\left\langle H^{c}\right\rangle_{t}\right)+\left(e^{x}-1\right) \star \mu\right) \frac{\partial \psi}{\partial y} \\
& +\frac{1}{2} \sigma^{2} \frac{\partial^{2} \psi}{\partial y^{2}} \leq 0 \text { on } S,
\end{aligned}
$$

respectively. We try a solution $\psi$ of the form

$$
\psi(y)=F(z), \quad \text { where } z=\pi y
$$


Equations (3.2) and (3.3) become

$$
\begin{gathered}
\pi F^{\prime}(z) \geq \pi \quad \text { or } \quad F^{\prime}(z) \geq 1, \\
A F(z)=-\rho F(z)+\left(\alpha t+C(\varphi)_{t}+\frac{1}{2}\left(\sigma^{2}+\left\langle H^{c}\right\rangle_{t}\right)+\left(e^{x}-1\right) \star \mu\right) \pi F^{\prime}(z) \\
+\frac{1}{2} \sigma^{2} \pi^{2} F^{\prime \prime}(z) \\
=-\rho F(z)+\kappa F^{\prime}(z)+\beta F^{\prime \prime}(z)<0,
\end{gathered}
$$

where

$$
\begin{aligned}
& \kappa=\left(\alpha t+C(\varphi)_{t}+\frac{1}{2}\left(\sigma^{2}+\left\langle H^{c}\right\rangle_{t}\right)+\left(e^{x}-1\right) \star \mu\right) \pi, \\
& \beta=\frac{1}{2} \sigma^{2} \pi^{2} .
\end{aligned}
$$

Inequality (3.7) is similar to inequality (3.11) in Lungu and Øksendal [10]. The major difference is that in Lungu and Øksendal [10] the coefficients are constants whereas in our case the coefficient $\kappa$ is not a constant. Since $\kappa(t, z)$ is a process with jumps, the general solution of

$$
\beta F^{\prime \prime}(z)+\kappa F^{\prime}(z)-\rho F(z)=0
$$

is elusive. We still try to explore the behavior of the solution after fixing the value of $t$; that is, we want to see the general behavior of the solution with respect to $z$.

\subsection{Examples}

\subsubsection{Example 1 ( $\kappa$ Constant with respect to $z$ )}

The case $\kappa$ is a constant that reduces to the problem in Lungu and Øksendal [10]. The auxiliary equation for (3.9) is

$$
\beta m^{2}+\kappa m-\rho=0
$$

The solutions are

$$
m_{1,2}=\frac{-\kappa \pm \sqrt{\kappa^{2}-4 \rho \beta}}{2 \beta} .
$$

Let us suppose the nonintervention region $B$ to be of the form

$$
B=\left\{z ; 0<z<z^{*}\right\}
$$


for some $z^{*}>0$. From (2.21) and (2.22), we try a solution of the form

$$
F(z)= \begin{cases}z+G, & \text { for } z \geq z_{* \prime} \\ \alpha_{1} e^{m_{1} z}+\alpha_{2} e^{m_{2} z}, & 0<z<z_{* \prime}\end{cases}
$$

for $G \in \mathbb{R}$. We want to determine parameters $\alpha_{1}, \alpha_{2}, G$, and $z_{*}$ such that $F$ becomes a $C^{2}$ at $z=z_{*}$. Using the continuity and differentiability of $F(z)$ at $z=z_{*}$ and taking $\alpha=\alpha_{1}=-\alpha_{2}$, we obtain

$$
\begin{aligned}
z_{*} & =\frac{2 \ln \left[m_{2} / m_{1}\right]}{\left(m_{1}-m_{2}\right)}>0, \\
\alpha & =\left(m_{1} e^{m_{1} z_{*}}+m_{2} e^{m_{2} z_{*}}\right)^{-1}, \\
G & =\alpha\left(e^{m_{1} z_{*}}-e^{m_{2} z_{*}}\right)-z_{*} .
\end{aligned}
$$

With this choice of parameters, all the conditions of Theorem 2.2 are satisfied, and we have

$$
\Phi(s, y)= \begin{cases}\alpha_{1} e^{-\rho s}\left(e^{m_{1} z}+e^{m_{2} z}\right), & \text { for } 0 \leq z<z_{*} \\ e^{-\rho s}(z+G), & z_{*} \leq z\end{cases}
$$

Similarly, as discussed in Lungu and Øksendal [10], the optimal strategy is obtained by doing nothing as long as $Y(t) \in\left(0, z_{*}\right)$ (i.e., $Y(t) \in B$ ) and to harvest a total amount $\gamma^{*}=\hat{\gamma}$ of the reflected process $Y \hat{r}$ in the direction of $-\pi$.

\subsubsection{Example 2 (When $\kappa$ Is Not a Constant)}

We now look at a more general case corresponding to $\kappa$ not a constant. Suppose that we can write $F(z)$ as

$$
F(z)=V(z) \exp \left(-\frac{1}{2} \int \frac{\kappa}{\beta} d z\right)
$$

then (3.9) can be transformed into its canonical form given by

$$
V^{\prime \prime}(z)+\eta(z) V(z)=0,
$$

where

$$
\eta=-\frac{\rho}{\beta}-\frac{1}{4}\left(\frac{\kappa}{\beta}\right)^{2}-\frac{1}{4}\left(\frac{\kappa}{\beta}\right)_{z}^{\prime}
$$

and the jumps are embedded in $\eta(z)$. 
To solve (3.17), we use the jump transfer matrix method [14]. The auxiliary equation of $(3.17)$ is

$$
m^{2}+\eta(z)=0
$$

with solutions

$$
m_{1}=i m(z), \quad m_{2}=-i m(z), \quad \text { where } m(z)=\sqrt{\eta(z)} .
$$

The general solution for $V(z)$ can be written as

$$
V(z)=h_{1}(z) \exp (i m z)+h_{2}(z) \exp (-i m z)
$$

where $h_{1}(z)$ and $h_{2}(z)$ are functions to be determined. The solution (3.21) can be expressed as

$$
V(z)=\exp [\Phi(z)]^{t} \mathbf{H}(z)
$$

where

$$
\mathbf{H}(\mathrm{z})=\left[\begin{array}{l}
h_{1}(z) \\
h_{2}(z)
\end{array}\right], \quad \Phi(z)=\left[\begin{array}{c}
i m(z) \\
-i m(z)
\end{array}\right]
$$

and the superscript $t$ denotes transpose of a matrix. $\mathbf{F}$ is the solution of the equation

$$
d \mathbf{H}(z)=\mathbf{U}(z) \mathbf{H}(z) d z,
$$

where

$$
\begin{array}{cc}
\mathbf{U}=-z \mathbf{K}^{\prime}(z)-\exp [-z \mathbf{K}(z)]\left[\mathbf{D}(z)^{-1} \mathbf{C}(z) \mathbf{K}^{\prime}(z) \exp [z \mathbf{K}(z)]\right] & \mathbf{C}(z)=\left[(p-1) m_{q}^{p-2}\right]_{n \times n^{\prime}} \\
\mathbf{K}(z)=\left[m_{p}(z) \delta_{p q}\right]_{n \times n^{\prime}} & \left.\mathbf{K}^{\prime}(x)=\left[m^{\prime}(z)_{q}^{p-1}(z)\right]_{n \times n^{\prime}}(z) \delta_{p q}\right]_{n \times n}
\end{array}
$$

(cf. [14]).

Clearly, for the case corresponding to $n=2$, the matrices (3.26) are given by

$$
\begin{gathered}
\mathbf{C}=\left(\begin{array}{ll}
0 & 0 \\
1 & 1
\end{array}\right), \quad \mathbf{D}=\left(\begin{array}{cc}
1 & 1 \\
m_{1} & m_{2}
\end{array}\right), \\
\mathbf{K}=\left(\begin{array}{cc}
m_{1} & 0 \\
0 & m_{2}
\end{array}\right), \quad \text { hence } \mathbf{K}^{\prime}=\left(\begin{array}{cc}
m_{1}^{\prime} & 0 \\
0 & m_{2}^{\prime}
\end{array}\right) .
\end{gathered}
$$


International Journal of Stochastic Analysis

Using power series expansion for exponential square matrices and truncating the expansion at second-order terms, we have

$$
\begin{aligned}
\exp [z \mathbf{K}] & =\exp \left[\left(\begin{array}{cc}
z m_{1} & 0 \\
0 & z m_{2}
\end{array}\right)\right] \\
& =\left(\begin{array}{ll}
1 & 0 \\
0 & 1
\end{array}\right)+\left(\begin{array}{cc}
z m_{1} & 0 \\
0 & z k_{2}
\end{array}\right)+O(2) \text { terms } \\
& =\left(\begin{array}{cc}
1+z m_{1} & 0 \\
0 & 1+z m_{2}
\end{array}\right)+O(2) \text { terms. }
\end{aligned}
$$

Similarly,

$$
\exp [-z \mathbf{K}]=\left(\begin{array}{cc}
1-z m_{1} & 0 \\
0 & 1-z m_{2}
\end{array}\right)
$$

From (3.25) and (3.28), we obtain an approximation for $\mathbf{U}(z)$ as

$$
\mathbf{U}(z)=\left[\begin{array}{cc}
-\left(z+\frac{1}{m_{1}-m_{2}}\right) m_{1}^{\prime} & \frac{m_{2}^{\prime}}{m_{2}-m_{1}} \exp \left[-z\left(m_{1}-m_{2}\right)\right] \\
\frac{m_{1}^{\prime}}{m_{1}-m_{2}} \exp \left[+z\left(m_{1}-m_{2}\right)\right] & -\left(z+\frac{1}{m_{2}-m_{1}}\right) m_{2}^{\prime}
\end{array}\right]
$$

From (3.19) and (3.20), we obtain

$$
\mathbf{V}(z)=\frac{m^{\prime}(z)}{2 m(z)}\left[\begin{array}{cc}
-1+i 2 m(z) z & \exp [i 2 z m(z)] \\
\exp [-2 i z m(z)] & -1-i 2 m(z) z
\end{array}\right]
$$

The jump transfer matrix from region 1 to region 2 across the interface $z=\varsigma$ in our case takes the form

$$
\mathbf{Q}_{1 \rightarrow 2}=\left[\begin{array}{ll}
\frac{m_{1}+m_{2}}{2 m_{2}} e^{+i \zeta\left(m_{2}-m_{1}\right)} & \frac{m_{2}-m_{1}}{2 m_{2}} e^{+i \zeta\left(m_{1}+m_{2}\right)} \\
\frac{m_{2}-m_{1}}{2 m_{2}} e^{-i \zeta\left(m_{1}+m_{2}\right)} & \frac{m_{2}+m_{1}}{2 m_{2}} e^{-i \zeta\left(m_{2}-m_{1}\right)}
\end{array}\right]
$$


This jump transfer matrix over singularities given by $\mathbf{Q}_{\varsigma^{-} \delta z \rightarrow \varsigma^{+} \delta z}$ is simplified as in [14] to

$$
\begin{aligned}
& \mathbf{Q}_{\varsigma^{-\delta z \rightarrow \varsigma+\delta z}}=\left[\begin{array}{cc}
\frac{1+i}{2} & \frac{1-i}{2} \\
\frac{1-i}{2} & \frac{1+i}{2}
\end{array}\right] \text { type A, } \\
& \mathbf{Q}_{\varsigma^{-\delta z \rightarrow \varsigma+\delta z}}=\left[\begin{array}{cc}
\frac{1-i}{2} & \frac{1+i}{2} \\
\frac{1+i}{2} & \frac{1-i}{2}
\end{array}\right] \quad \text { type B, } \\
& \mathbf{Q}_{\delta^{-\delta z \rightarrow \varsigma^{+} \delta z}}=\left[\begin{array}{ll}
1 & 0 \\
0 & 1
\end{array}\right] \quad \text { type C. }
\end{aligned}
$$

The reader is referred to [14] and the references therein for details regarding classification of singularities.

\subsubsection{Analysis of the Results}

Let $z_{0}=0$ and our solutions (3.21) have jumps at points $z_{1}, z_{2}, z_{3}, \ldots, z_{n}$, and we define

$$
\begin{gathered}
W_{j}=\left\{z: z_{j-1}<z<z_{j}\right\} \quad \text { for } i=1,2,3, \ldots, n, \\
B_{j}=\left\{z: z_{j-1}<z<z_{* j}\right\}
\end{gathered}
$$

for some $z_{* i}$, and we define

$$
B=\bigcup_{j=1}^{n} B_{j}
$$

For $z \in W_{i}$, we define

$$
\begin{aligned}
F_{j}(z) & = \begin{cases}z+G_{j}, & \text { for } z \geq z_{j *}, \\
h_{j 1}(z) e^{m_{j 1} z}+h_{j 2} e^{m_{j 2} z}, & 0<z<z_{j * \prime}\end{cases} \\
F & =\left\{F_{0}, F_{1}, F_{2}, \ldots, F_{n}\right\}, \\
\Phi(s, z)_{j} & = \begin{cases}h_{j}(z) e^{-\rho s}\left(e^{m_{j 1} z}+e^{m_{j 2} z}\right), & \text { for } 0 \leq z<z_{j * \prime} \\
e^{-\rho s}\left(z+G_{j}\right), & z_{j *} \leq z,\end{cases} \\
\Phi(s, z) & =\left\{\Phi_{1}(s, z), \Phi_{2}(s, z), \ldots, \Phi_{n}(s, z)\right\} .
\end{aligned}
$$

\subsubsection{Conjecture}

The optimal strategy is achieved by doing nothing during jumps and as long as $Y(t) \in B_{j}$ but to harvest according to local time $\gamma_{j}^{*}=\hat{\gamma}$ at the boundary $\partial B_{j}$.

Remarks. In each nonintervention region $B_{j}, Y^{\widehat{\gamma}}$ is a reflected process at $\partial B_{j}$, since, as $\Upsilon^{\hat{\gamma}}$ hits the boundary, a certain amount is harvested thereby forcing the process to go below $z_{j *}$. 


\section{Conclusion}

Most of the conditions in Theorem 2.2 are extensions from Lungu and Øksendal [10] with exception of condition (2.5), that is,

$$
\begin{aligned}
\frac{\partial \phi}{\partial y}\left(t, Y^{\gamma}(s)\right)\left(\alpha d t+d C(\phi)_{t}+\frac{1}{2} d\left\langle H^{c}\right\rangle+\int_{-\infty}^{\infty}\left(e^{y}-1-\phi(y) d \mu\right)\right) \\
-\left(\alpha t+C(\phi)_{t}+\frac{1}{2}\left(\sigma^{2}+\left\langle H^{c}\right\rangle_{t}^{\prime}\right)+\int_{-\infty}^{\infty}\left(e^{y}-1-\phi(y)\right) d \mu\right) d t \leq 0
\end{aligned}
$$

We note that this condition is achieved if $\partial \phi / \partial y$ is small. It is found under additional condition (2.27) that our problem can be reduced to a second-order differential equation similar to that in Lungu and Øksendal [10] though with some jumps. What is observed is that if the investment process is being modeled by a semimartingale in general, optimal value function $\Phi(s, y)$ and the optimal dividend strategy can be found if the rate of change of the value function $\phi(s, y)$ with respect to the investment process itself, that is, $\partial \phi / \partial y$, is small enough. In other words, $\phi(s, y)$ should not be too sensitive to variations in investments. Our results further show that the general solution to this problem is still elusive.

\section{References}

[1] M. Miller and Modigilliani, "Dividend policy, growth and valuation of shares," Journal of Bussiness, vol. 34, pp. 411-433, 1961.

[2] M. I. Taksar, "Optimal risk and dividend distribution control models for an insurance company," Mathematical Methods of Operations Research, vol. 51, no. 1, pp. 1-42, 2000.

[3] S. Asmussen, B. Højgaard, and M. Taksar, "Optimal risk control and dividend distribution policies. Example of excess-of loss reinsurance for an insurance corporation," Finance and Stochastics, vol. 4, no. 3, pp. 299-324, 2000.

[4] S. Asmussen and M. Taksar, "Controlled diffusion models for optimal dividend pay-out," Insurance: Mathematics \& Economics, vol. 20, no. 1, pp. 1-15, 1997.

[5] B. Højgaard and M. Taksar, "Controlling risk exposure and dividends payout schemes: insurance company example," Mathematical Finance, vol. 9, no. 2, pp. 153-182, 1999.

[6] B. Højgaard and M. Taksar, "Optimal risk control for a large corporation in the presence of returns on investments," Finance and Stochastics, vol. 5, no. 4, pp. 527-547, 2001.

[7] R. Radner and L. Shepp, "Risk vs profit potential: a model for corporate strategy," Journal of Economic Dynamics and Control, vol. 20, pp. 1373-1393, 1996.

[8] T. Choulli, M. Taksar, and X. Y. Zhou, "Excess-of-loss reinsurance for a company with debt liability and constraints on risk reduction," Quantitative Finance, vol. 1, no. 6, pp. 573-596, 2001.

[9] M. Jeanblanc-Picqué and A. N. Shiryaev, "Optimization of the flow of dividends," Russian Mathematical Survey, vol. 50, pp. 25-46, 1995.

[10] E. Lungu and B. Øksendal, "Optimal harvesting from interacting populations in a stochastic environment," Bernoulli, vol. 7, no. 3, pp. 527-539, 2001.

[11] P. Protter, Stochastic Integration and Differential Equations, Springer, New York, NY, USA, 2nd edition, 1992.

[12] A. N. Shiryaev, Essentials of Stochastic Finance, vol. 3 of Advanced Series on Statistical Science E Applied Probability, World Scientific Publishing, River Edge, NJ, USA, 1999.

[13] H. Buhlman, F. Delbaen, P. Embrechts, and N. Albert, "No arbitrage, change of measure and esscher transforms," CW Quarterly, vol. 9, pp. 291-317, 1969.

[14] S. Khorasani and A. Adibi, "Analytical solution of linear ordinary differential equations by differential transfer matrix method," Electronic Journal of Differential Equations, vol. 79, pp. 1-18, 2003. 


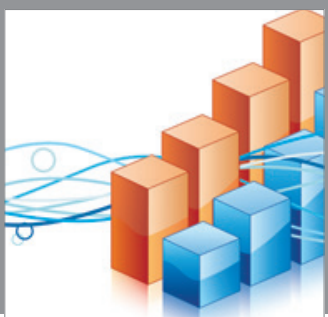

Advances in

Operations Research

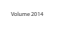

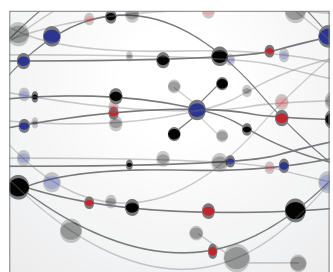

\section{The Scientific} World Journal
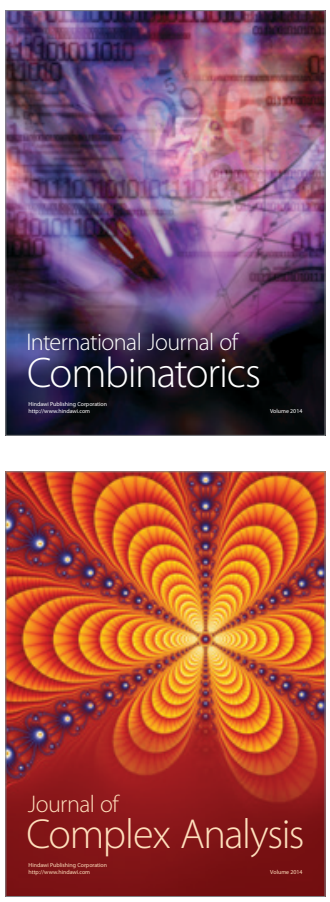

International Journal of

Mathematics and

Mathematical

Sciences
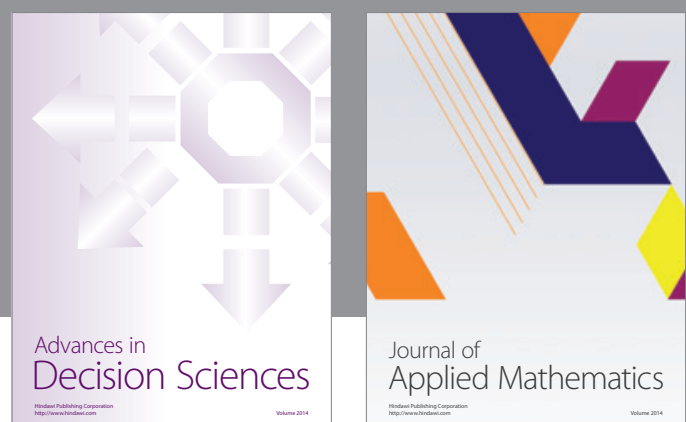

Journal of

Applied Mathematics
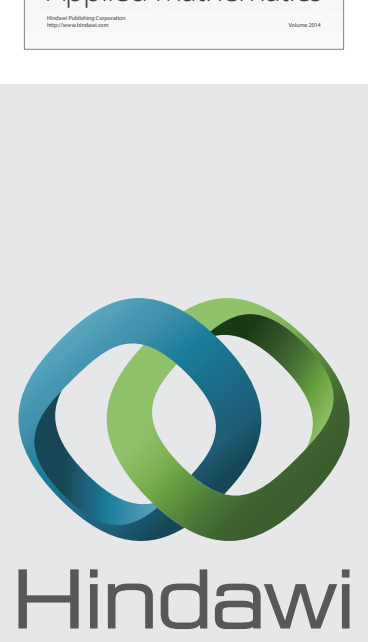

Submit your manuscripts at http://www.hindawi.com
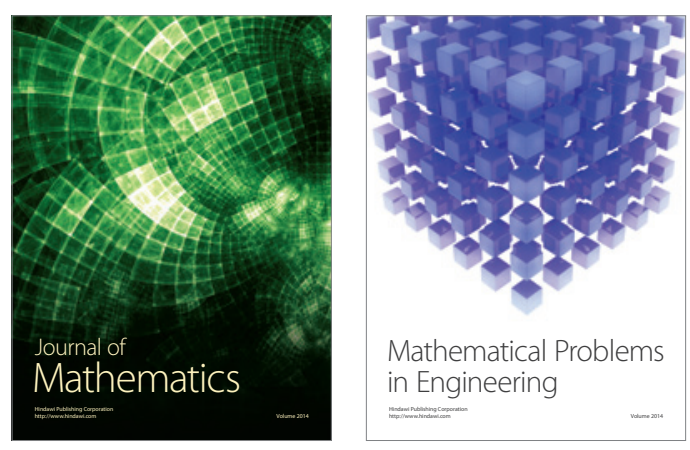

Mathematical Problems in Engineering
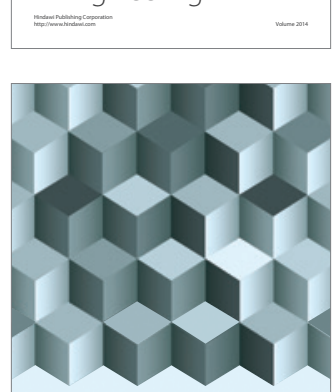

Journal of

Function Spaces
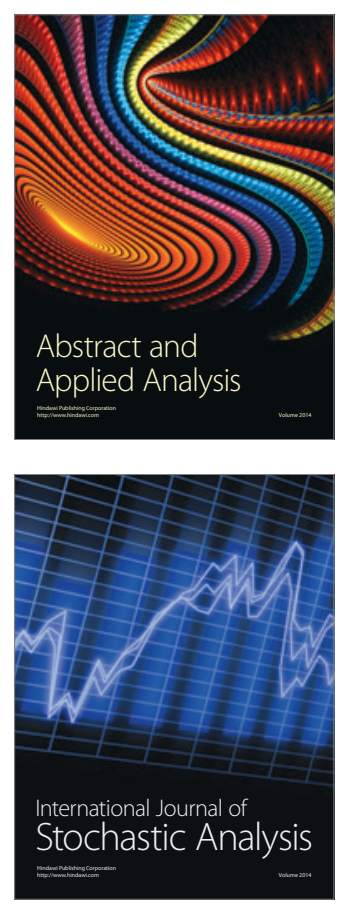

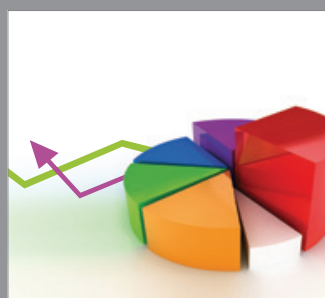

ournal of

Probability and Statistics

Promensencen
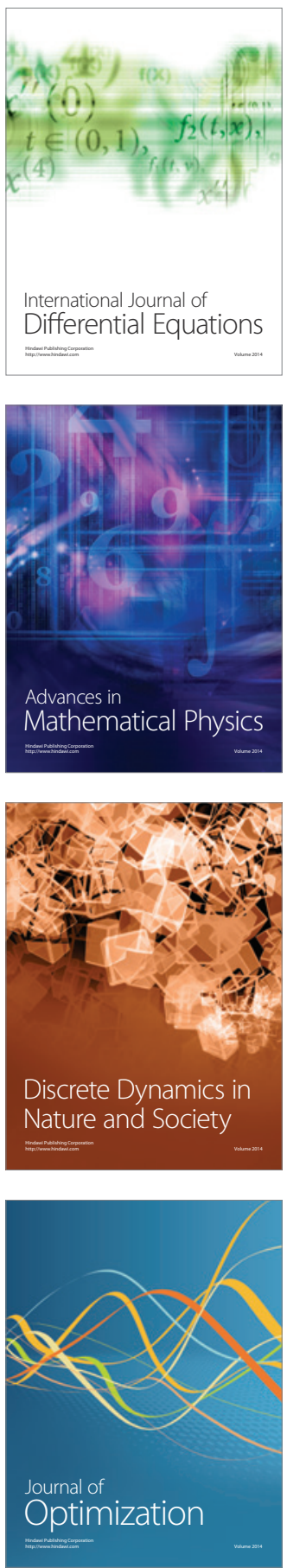